\title{
A chronic pneumothorax and fitness to fly
}

\author{
Graeme P Currie, Ann-Maree Kennedy, Edward Paterson, Stephen J Watt
}

Thorax 2007;62:187-189. doi: 10.1136/thx.2004.035055

According to Boyle's law, as the pressure falls, the volume of gas rises in an inversely proportional manner. This means that during an aircraft flight, the volume of trapped air in gas filled body chambers will increase. As a consequence, it is fairly well established that individuals with an untreated pneumothorax should not participate in commercial flying due to the risk of it enlarging and the possible development of tension. However, whether this also applies to individuals who have a longstanding, clinically stable pneumothorax is uncertain. The following article describes two adult patients each with a chronic pneumothorax who asked whether they would be fit to fly in an aircraft. We outline their histories and subsequent evaluation which consisted of clinical assessment, computed tomographic imaging, a hypoxic challenge test and exposure to a hypoxic hypobaric environment in a decompression chamber.

A dvice for individuals with respiratory disorders concerning fitness to fly in commercial aircraft has previously been lacking clear methodology and consistency. ${ }^{1}$ As a consequence, the British Thoracic Society Standards of Care Committee (London, UK) has developed evidence-based guidelines to advise and manage patients with respiratory diseases who wish to fly. ${ }^{2}$ Without definitive intervention, individuals with an untreated pneumothorax should not participate in commercial flying. Whether this advice applies to patients with a long-standing pneumothorax is uncertain as there is a paucity of literature guiding clinicians and patients in this respect. We report two adult patients with persistent pneumothoraces who underwent a normobaric hypoxic challenge test to identify significant desaturation. ${ }^{3}$ This involved patients breathing 15\% oxygen at sea level to mimic the reduced inspiratory oxygen pressure to which they would be exposed during a typical commercial flight. Only after this was performed with an acceptable fall in oxygen saturation did the individuals proceed to cabin altitude simulation in a hypobaric chamber (hypobaric hypoxia), where full cardiorespiratory resuscitation facilities were available. The pre-flight assessments in both patients proved satisfactory, and one of them (case 1) has successfully flown on commercial transatlantic flights.

\section{CASE 1}

A 36-year-old male smoker (15 pack years) was admitted to hospital with progressive respiratory failure secondary to varicella pneumonitis with superadded staphylococcal infection. Although intubated and mechanically ventilated, his progress was complicated by adult respiratory distress syndrome and the development of bilateral pneumothoraces, which required multiple chest drain insertions. Two months after discharge, he was readmitted with a further right pneumothorax, which was treated with a chest drain.

Subsequent follow-up showed a persistent loculated pneumothorax in the left upper zone. At clinic review 4 years after initial presentation, he enquired whether he was fit to fly.
Forced expiratory volume in $1 \mathrm{~s}$ was $2.7 \mathrm{l}$, forced vital capacity was $3.3 \mathrm{l}$ (predicted values 4.1 and $5.0 \mathrm{l}$, respectively), carbon monoxide transfer factor was $5.5 \mathrm{mmol} / \mathrm{min} / \mathrm{kPa}$ (predicted $11.5 \mathrm{mmol} / \mathrm{min} / \mathrm{kPa}$ ) and resting oxygen saturation was $98 \%$. Computed tomography of the chest showed a small left pneumothorax (estimated volume $110 \mathrm{ml}$ ) and thickened pleura overlying the lung surface (fig 1). During a hypoxic challenge test breathing $15 \%$ oxygen, his saturation fell to $90 \%$. An altitude chamber trial was then performed involving a simulated ascent to 8000 feet $(2438 \mathrm{~m})$ over $15 \mathrm{~min}$, at 8000 feet $(2438 \mathrm{~m})$ for $20 \mathrm{~min}$ and descent over $10 \mathrm{~min}$. During altitude simulation, the patient remained asymptomatic and oxygen saturation fell to $90 \%$. As a consequence of the assessment, he was considered fit to fly and has completed over a dozen flights, mostly transatlantic, without difficulty.

\section{CASE 2}

A 31-year-old female smoker (20 pack years) initially presented to cardiothoracic surgeons with a spontaneous right-sided pneumothorax. She had sustained two previous spontaneous right-sided pneumothoraces, each requiring treatment by chest drain insertion. She successfully underwent a partial parietal pleurectomy with stapling of bullae and was asymptomatic on discharge.

She subsequently developed three separate left pneumothoraces, each of which resolved without intervention. After referral for surgery, she proceeded to video-assisted thoracoscopic (VATS) stapling of bullae and talc pleurodesis. Over the next 18 months, she developed three further spontaneous right pneumothoraces and was referred back to the cardiothoracic surgeons and underwent a right VATS pleurodesis. Her hospital stay was prolonged because of difficulties with lung re-expansion post-operatively, and at the time of discharge she continued to have a residual right apical pneumothorax.

The following year the patient enquired as to whether she was fit to fly. Spirometry was within the normal range with a forced expiratory volume in $1 \mathrm{~s}$ of $2.65 \mathrm{l}$ and a forced vital capacity of $3.3 \mathrm{l}$ with carbon monoxide transfer factor being mildly impaired at $6.4 \mathrm{mmol} / \mathrm{min} / \mathrm{kPa}$ (predicted $9.5 \mathrm{mmol} /$ $\mathrm{min} / \mathrm{kPa}$ ). Computed tomography imaging of her chest showed emphysematous changes and a loculated right-sided pneumothorax (estimated volume $250 \mathrm{ml}$ ) with thickened pleura over the lung surface (fig 2). During a hypoxic challenge test breathing $15 \%$ oxygen, her saturation fell from $98 \%$ at room air to $93 \%$. She went on to have an altitude chamber test involving simulated ascent to 8000 feet $(2438 \mathrm{~m})$ with ascent and descent over $15 \mathrm{~min}$. This was tolerated without symptoms and her oxygen saturation fell to a minimum of $92 \%$. She was therefore considered fit to fly in commercial aircraft.

\section{DISCUSSION}

Air travel provides an accessible and inexpensive transport modality for an increasing number of people and therefore

Abbreviation: VATS, video-assisted thoracoscopy 


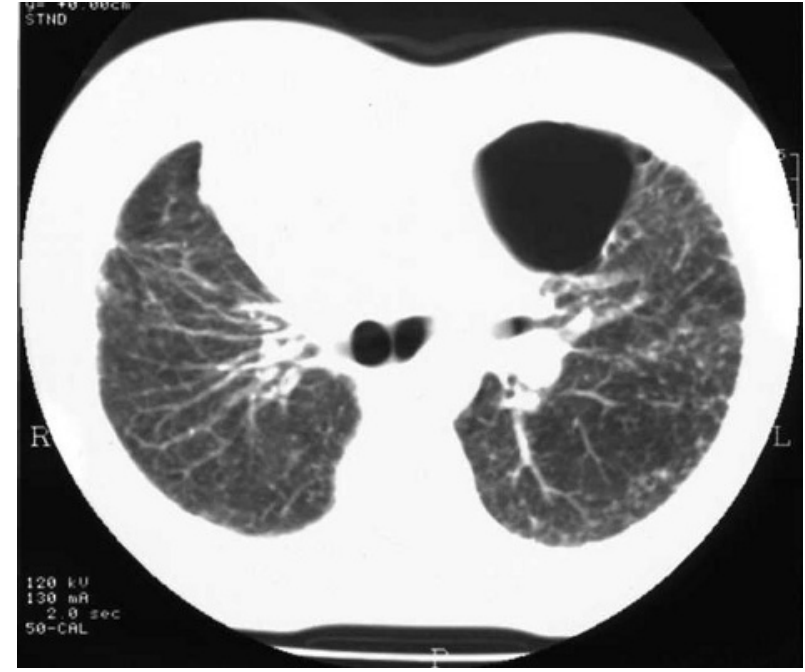

Figure 1 Computed tomographic image showing the loculated left-sided pneumothorax in case 1.

includes individuals of varying ages (even the extremes) and with a variety of medical problems. Pneumothoraces can be primary-commonly affecting healthy slim young males-or secondary. Their development has implications for those exposed to changes in atmospheric pressure-for example, during diving ${ }^{4}$ and flying. The precise mechanism surrounding the development of a pneumothorax is unclear, but rupture of surface blebs or bullae has been suggested as a possible mechanism. Although most episodes of spontaneous pneumothorax are not associated with exertion or activity, both diving and altitude exposure have been identified as provocative factors. The expansion of gas in the lung during reduction in pressure is assumed to lead to lung rupture. There are no data outlining the risks associated with air travel in patients with a closed pneumothorax. However, a case report describes a diver who undertook approximately 80 dives with a chronic pneumothorax without difficulty, but who did become breathless during a 5 -h flight. ${ }^{5}$

The British Thoracic Society guidelines ${ }^{2}$ suggest that individuals with a closed pneumothorax should be advised to avoid commercial flights. However, an updated recommendation now suggests that patients who have had a pneumothorax must have a chest radiograph confirming its resolution with at least 7 days elapsing thereafter before a flight. In individuals with a traumatic pneumothorax, it is suggested that before flying 2 weeks should elapse following full radiographic resolution. ${ }^{6}$ Several studies have shown that the risk of recurrence of pneumothorax is reduced by surgical intervention irrespective of whether this involves thoracotomy or VATS procedure.

According to Boyle's law, as the pressure falls during ascent, an inversely proportional rise in gas volume occurs. This in turn causes expansion of air in gas-filled bodily chambers such as the middle ear, sinuses, intestines, non-communicating lung bullae and a closed pneumothorax. Therefore, airline passengers with a closed pneumothorax may experience difficulties due to gas expansion during ascent, and risk developing a tension pneumothorax and possible cardiovascular collapse. Moreover, even in healthy people, the partial pressure of oxygen in arterial blood falls with altitude, which further compounds any respiratory difficulties encountered with expansion of a pneumothorax. For example, at 8000 feet $(2438 \mathrm{~m})$, the partial pressure of oxygen in arterial blood falls to the equivalent of breathing approximately 15\% oxygen at sea level. Aircraft tend

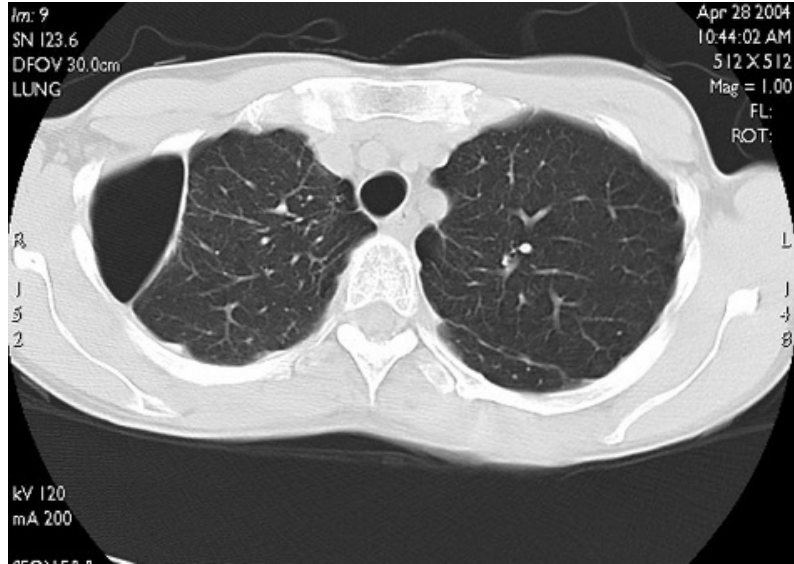

Figure 2 Computed tomographic image showing the loculated right-sided pneumothorax in case 2 .

to be noisy with the inevitable difficulty in diagnosing a pneumothorax with certainty, while the availability of necessary medical equipment for its treatment is largely determined by the time required for the aircraft to land.

The individuals in both cases had a chronic pneumothorax, which had been stable for $>1$ year. This condition has generally precluded air travel because of concerns regarding development of a tension pneumothorax. However, we felt that the risk of recurrence with altitude exposure was small. Indeed, in case 1 there had been a long period of stability and in case 2 bilateral surgical intervention had been performed. Moreover, in both cases computed tomography of the chest suggested the presence of thickened pleura. Before exposing both individuals to the dangers associated with a simulated flight in a hypobaric chamber, a hypoxic challenge test was arranged. ${ }^{3}$ It is relevant to point out that neither resting oxygen saturation nor lung function is a reliable predictor of response to hypoxic challenge testing. ${ }^{8}$ For example, Seccombe et al ${ }^{9}$ evaluated patients with chronic obstructive pulmonary disease and interstitial lung disease with an oxygen saturation of $96 \%$ at sea level. Levels of oxygenation fell to below $90 \%$ when cabin altitude was simulated, with an even further desaturation during minimal exercise.

In the knowledge that the reduced inspired partial pressure of oxygen did not result in unacceptable hypoxaemia, we proceeded to hypobaric hypoxia simulation. The size of the chronic pneumothorax in both cases was relatively small and as a consequence, a minimal effect of volume expansion was expected. Indeed, it is uncertain what volume of air in a pneumothorax can be tolerated and because expansion of a large pneumothorax can undoubtedly result in development of tension, careful assessment of the volume using computed tomography of the chest was important. Subsequent satisfactory completion of a simulated altitude exposure under controlled conditions provided confirmation that volume expansion did not result in symptoms or greater hypoxaemia. However, common sense suggests that satisfactory completion of a hypoxic challenge test and hypobaric chamber assessment cannot eliminate the risks associated with flights in the future.

We conclude that some patients with a closed chronic pneumothorax can fly without adverse consequences. The risk, however, should be determined after thorough assessment incorporating computed tomography of the chest, a hypoxic challenge test and simulation of flying at altitude in a decompression chamber with close monitoring of symptoms and oxygen saturation. Whether the suitability of our proposed 
assessment and subsequent findings can be extrapolated to include other patients with larger pneumothoraces requires further evaluation.

\section{Authors' affiliations}

G P Currie, A-M Kennedy, E Paterson, Department of Respiratory Medicine, Aberdeen Royal Infirmary, Foresterhill, Aberdeen, Scotland, UK S J Watt, Department of Environmental and Occupational Medicine, University of Aberdeen, Aberdeen, Scotland, UK

Competing interests: None.

Correspondence to: $\operatorname{Dr}$ G P Currie, Chest Clinic C, Aberdeen Royal Infirmary, Foresterhill, Aberdeen AB25 2ZN, Scotland, UK; graeme. currie@nhs.net

Received 17 September 2004

Accepted 17 January 2005

\section{REFERENCES}

1 Coker RK, Partridge MR. Assessing the risk of hypoxia in flight: the need for more rational guidelines. Eur Respir J 2000;15:128-30.

2 Coker RK, Boldy DAR, Buchdahl R, et al. Managing passengers with respiratory disease planning air travel: British Thoracic Society recommendations. Thorax 2002;57:289-304

3 Gong $\mathbf{H}$ Jr, Tashkin DP, Lee EY, et al. Hypoxia-altitude simulation test. Evaluation of patients with chronic airway obstruction. Am Rev Respir Dis 1984;130:980-6.

4 Godden D, Currie GP, Denison D, et al. Guidelines on respiratory aspects of fitness for diving. Thorax 2003:58:3-13.

5 Ziser A, Vaananen A, Melamed Y. Diving and chronic spontaneous pneumothorax. Chest 1985;87:264-5.

6 British Thoracic Society. http://www.brit-thoracic.org.uk/c2/uploads/ flightrevision04.pdf.

7 Sedrakyan A, van der Meulen J, Lewsey J, et al. Video assisted thoracic surgery for treatment of pneumothorax and lung resections: systematic review of randomised clinical trials. BMJ 2004;329:1008.

8 Robson AG, Hartung TK, Innes JA. Laboratory assessment of fitness to fly in patients with lung disease: a practical approach. Eur Respir J 2000;16:214-19.

9 Seccombe LM, Kelly PT, Wong CK, et al. Effect of simulated commercial flight on oxygenation in patients with interstitial lung disease and chronic obstructive pulmonary disease. Thorax 2004;59:966-70.

\section{bmjupdates+}

bmjupdates+ is a unique and free alerting service, designed to keep you up to date with the medical literature that is truly important to your practice.

bmjupdates+ will alert you to important new research and will provide you with the best new evidence concerning important advances in health care, tailored to your medical interests and time demands.

Where does the information come from?

bmiupdates+ applies an expert critical appraisal filter to over 100 top medical journals

A panel of over 2000 physicians find the few 'must read' studies for each area of clinical interest

Sign up to receive your tailored email alerts, searching access and more...

www.bmjupdates.com 